\title{
Polycystic ovary syndrome and its impact on women's quality of life: More than just an endocrine disorder
}

\author{
Christine Brady \\ Shaymaa S Mousa \\ Shaker A Mousa \\ The Pharmaceutical Research \\ Institute, Albany College of Pharmacy \\ and Health Sciences, Albany, NY, USA
}

Correspondence: Shaker A Mousa Executive VP and Chairman of the Pharmaceutical Research Institute, The Albany College of Pharmacy,

One Discovery Drive, Rensselaer, NY 12144 , USA

Tel + I 5186947397

Fax +I 5186947567

Emailshaker.mousa@acphs.edu

\begin{abstract}
In the past, polycystic ovary syndrome has been looked at primarily as an endocrine disorder. Studies now show that polycystic ovary syndrome is a metabolic, hormonal, and psychosocial disorder that impacts a patient's quality of life. It is extremely important to holistically treat these patients early on to help them deal with the emotional stress that is often overlooked with polycystic ovary syndrome. Early diagnosis and long term management can help control polycystic ovary syndrome so that women can still live a healthy active life and avoid long-term complications such as metabolic syndrome and cardiovascular diseases.

Keywords: polycystic ovary syndrome, quality of life, sexual satisfaction, infertility, psychological distress, hirsutism, metabolic syndrome
\end{abstract}

\section{Background}

Polycystic ovary syndrome (PCOS) is one of the most common endocrine disorders in women during their reproductive years ${ }^{1}$ with an estimated prevalence in 7-10 million American women. ${ }^{2}$ It affects $5 \%-10 \%$ of women in the developed world and is the most common endocrine disorder of women in their reproductive years ${ }^{3}$ PCOS is typically defined as hyperandrogenism associated with chronic anovulation in women without other underlying disease. ${ }^{3}$ At any age, PCOS can be devastating to women, especially during the reproductive years; PCOS is the leading cause of female infertility. ${ }^{4}$ Many women are often not immediately diagnosed with PCOS, and it is important for physicians to look for the hallmark signs of PCOS, such as menstrual cycle irregularity, hirsutism, infertility and a family history. ${ }^{4}$ Currently there is no consensus on diagnostic criteria for PCOS. The National Institutes of Health (NIH) consensus criteria consists of a minimal finding of: (i) menstrual irregularity due to olio- or anovulation, (ii) evidence of hyperandrogenism, whether clinical (hirsutism, acne, or male pattern balding) or biochemical (high serum androgen concentrations), (iii) exclusion of other causes of hyperandrogenism and menstrual irregularity (such as congenital adrenal hyperplasia, hyperprolactinemia, and androgen-secreting tumors). According to the Rotterdam consensus, a diagnosis of PCOS requires the presence of two of the following three conditions: (i) oligo- and/or anovulation, (ii) clinical and/or biochemical signs of hyperandrogenism (acne, hirsutism, alopecia), and (iii) polycystic ovaries and the exclusion of other etiologies., ${ }^{5,6}$ The AES (Androgen Excess Society) taskforce determined that the original NIH criteria should be accepted with some modifications from the Rotterdam consensus (the only difference between the Rotterdam and AES diagnostic criteria is that according to Rotterdam criteria, a patient with polycystic ovaries and oligo- or anovulation would be diagnosed with PCOS, while under AES criteria they would not). ${ }^{7}$ 
It is important to note that outside of these criteria, women with PCOS often experience other conditions that can affect their short- and long-term physical and mental health. Decreased quality of life from mood disturbances, decreased sexual satisfaction, weight gain, acne vulgaris, and alopecia have all been documented. ${ }^{8}$ PCOS may represent one of the largest groups of women at high risk for the development of early onset coronary heart disease. ${ }^{9}$ Although it is important to treat the short term disturbances for women, research shows that it is important to think about the future of women with PCOS, as many of them will develop metabolic syndrome..$^{10}$ Metabolic syndrome is a collection of cardiovascular disease risk factors associated with insulin resistance, dyslipidemia, hypertension, and central obesity. ${ }^{11}$ This review highlights the current status regarding several important aspects of PCOS.

\section{PCOS and quality of life in patients}

Research shows that both physical health consequences and the emotional impact of PCOS have been ignored..$^{12}$ PCOS and its influence on quality of life is an issue that needs to be taken seriously as this syndrome affects many women across the world. Measuring the quality of life of patients is often hard to do accurately. What one person may feel is a poorer quality of life may be very well suitable for another person. It is important to realize that although these scales and questionnaires have been well documented the information taken from them is often subjective. How do we define being satisfied with our quality of life? For one woman it may be having a family and being a mother, for another woman it may be having a successful career, or for some it may be both. Standardized questionnaires such as the Polycystic Ovary Syndrome Health-Related Quality of Life Questionnaire (PCOSQ), the Short-Form Health Survey-36 (SF-36), Symptom Check List-90, and Visual Analog Scales (VAS), along with control groups, are often used to try to overcome these barriers. ${ }^{8}, 13$ Simply asking a patient how they are feeling may not prompt them to truly express the nature of their feelings. Patients were formerly treated primarily on their symptoms of PCOS, but current studies have shown that patients need to be treated more holistically, as PCOS can affect a woman's mind, body, and her identity with herself as a woman.

Scales measuring quality of life are either generic or specific. Instruments that are illness specific provide more accurate information for that disease, while generic questionnaires such as the SF-36, although well validated, may lack in sensitivity as they are geared to measure health status across a wide variety of diseases..$^{14,15}$ The SF-36 scale leaves out important detrimental issues linked to PCOS patients such as infertility and hirsutism, two items that can heavily impact a woman's life. ${ }^{3}$ In 1998, the PCOSQ was created to specifically address these problems. This questionnaire focuses on the physical and emotional symptoms related to hirsutism, emotional impact of infertility, and physical symptoms associated with menstruation that can decrease a women's overall quality of life. ${ }^{3}$

Although the SF-36 may lack sensitivity, the use of the questionnaire has been well validated, especially its use in conjunction with the PCOSQ. A recent study shows that the PCOSQ has reasonable internal reliability, good test-retest reliability, good concurrent and discriminant validity, and a reasonable factor analysis; ${ }^{14}$ making PCOSQ a useful and promising tool for measuring health-related quality of life (HRQoL) in PCOS patients. ${ }^{3,16}$ Based on the research shown by these scales, it is accurate to say that PCOS is indeed more than an endocrine disorder and that the effects of the diseases can have emotionally and psychological impact. ${ }^{14}$ With this knowledge, it is justified to take a further look into the exact ways in which PCOS can affect a woman's total quality of life.

\section{Sexual satisfaction}

Although not all women (specifically adolescents) with PCOS may be sexually active, at some point in their lives they may be and sexual satisfaction may become important to them. Sexual satisfaction can play an important role, biologically and mentally, in a person's life, as well as in relationships.

To assess sexual satisfaction, VAS are used, ranging from 0-100 (with 0 being not at all affected, to 100 being very much affected). In one study, it was found that there was no statistical significance in the frequency of sexual intercourse or thoughts of sex by either the PCOS group or the control group; what did prove significant is that women with PCOS were shown to be significantly less satisfied with their sex life. ${ }^{13}$ Women with PCOS felt that their partners found them less attractive and felt that their partners were less satisfied with their sexual relationship. Women with PCOS who were obese, or not obese, according to VAS results, both reported being less satisfied with their sex life and both felt less sexually attractive. ${ }^{13}$

Studies evaluating sexual satisfaction can prove statistically significant, but should be looked at only as a general conclusion. A study comparing healthy controls to women with PCOS showed no differences in sociodemographic 
variables, finances, marriage, friends, and living conditions, leading researchers to believe that the differences in sexual satisfaction were due to the presence or absence of PCOS. ${ }^{13}$ It is important to take note that in this study, women in control groups did not rate their satisfaction at 100, showing that there may be other factors in women's lives that determine if their sex life is satisfying and that PCOS may not be the root cause of dissatisfaction.

\section{Infertility}

PCOS is the most common endocrine disorder causing female fertility regardless of ethnicity. ${ }^{13,17,18}$ Approximately $20 \%$ of couples in western society experience infertility. ${ }^{17}$ Infertility occurs due to the ovulatory disturbances in PCOS, ${ }^{19}$ and $100 \%$ of patients with PCOS suffer from ovulatory dysfunction according to the NIH 1990 criteria. ${ }^{20}$ Of this, $75 \%$ of women with PCOS will experience infertility. ${ }^{21}$

For some women, having children is an important part of the female identity. Not only may her quality of life be affected, her partner or family may be affected; it should be noted that the extent of infertility on quality of life varies according to socio-cultural factors, traditions, and religious beliefs. ${ }^{18}$ Infertility is often associated with divorce, low social status, lowered self-esteem, altered self-perception, and job dissatisfaction. ${ }^{22,23}$ PCOS may also trigger infertility by causing stress and other psychosocial factors including distress, depression, anxiety, sexual problems, marital, and social maladjustment, lost of control, and lowered self esteem. $^{17}$

Infertility does not simply become a concern to women with PCOS when they are trying to conceive, but recent studies have shown that it is a problem that even adolescent girls with PCOS are concerned about. If aware of infertility at a young age, women with PCOS may be inhibited emotionally and physically in establishing a long term relationship. In fact, one study reported a 3.4-fold incidence of concern using the Child Health Questionnaire-Child SelfReport Form (CHQ-CF-87).

The PCOSQ questionnaire is often used to assess the impact of infertility on a woman with PCOS quality of life. Research has shown that patients with PCOS had a significant negative impact on quality of life in many domains, including infertility, which had a $51 \%$ reduction in HRQoL compared to the control group. ${ }^{14}$ In this study, infertility had an alpha score above 0.90 , indicating excellent reliability. ${ }^{14}$ It is reassuring to know that researchers have an accurate tool to measure the quality of life in PCOS patients, but now the problem is how to directly treat these issues.
One noteworthy study showed the importance of a Patient Information Questionnaire (PIQ). ${ }^{19}$ This questionnaire asked patients several questions, one of them being how they would rate the information given to them about how to handle fertility issues with PCOS. A majority of patients (48\%) rated the information given poor, $31 \%$ fair, $18 \%$ good, $15 \%$ very good, $3 \%$ excellent in regards to fertility. Forty-seven percent of patients gained the most information from their physician, while $39 \%$ of information was gained from the Internet. ${ }^{19}$ These numbers prove an important point, establishing a way to give patients better information is critical. It can be estimated that $50 \%$ of PCOS patients will actively seek infertility services at some point in their lifetime. ${ }^{24}$ Further studies need to be performed to assess whether or not these patients who seek infertility services were better informed about fertility and had better quality of life, because they felt more knowledgeable about the syndrome.

What has not been studied is to what extent does infertility impact the quality of life. ${ }^{25}$ Studies have shown that it can indeed decrease a woman's quality of life, but more studies must be done to take into account different factors such as demographic features, ${ }^{18}$ previous treatment failure, ${ }^{26,27,28}$ and length of infertility. ${ }^{12,29}$ Another important point for consideration is that infertility can be brought on by other triggers such as stress and depression. Of women with PCOS who experience infertility, the incidence of infertility in lean women with PCOS may be less, ${ }^{21}$ showing that weight may be a factor of infertility. Keeping these co-morbidities under control may be the key in preventing infertility in some women.

\section{Psychological distress and emotional distress}

It has been well documented that women with PCOS have higher levels of psychological distress. ${ }^{30}$ Symptoms of PCOS such as obesity, hirsutism and infertility have a strong influence on the psychosocial experience of women. ${ }^{30}$ Additionally, women with PCOS have been found to have higher levels of depression and overall psychological morbidity and decreased quality of life in overall health and sex. . $^{13,31}$

With diagnosis of any disorder, disease, or syndrome it is reasonable to say that a patient may have anxiety. The causes of this added anxiety in life is most attributable to hirsutism, irregular menses, and infertility.

One study analyzed the incidence of mental distress in women with untreated PCOS using self reported measures to characterize PCOS patients at risk for psychiatric disease with regards to sociodemographic and clinical characteristics and to assess the impact of emotional distress on quality 
of life. ${ }^{32}$ To assess psychological distress, the German version of the SCL-90R was used to assess mental well being. This scale can be used for screening against cases of psychiatric/psychological illness. This five-point scale assesses symptoms in nine areas: somatization, obsessivecompulsive, interpersonal sensitivity, depression, anxiety, aggression, phobia, paranoid ideation, and psychoticism. The average of all 90 items gives a Global Severity Index (GSI) score that represents the overall level of distress in a patient. ${ }^{33}$ Additionally, the Positive Symptom Distress Index (PSDI) was measured to depict the intensity of distress, while the Positive Symptom Total (PST) was taken to show the total number of distress-inducing symptoms. ${ }^{32}$

Of the 143 patients in this study, $15.4 \%$ had a possible psychological disorder based on GSI scores greater than 63 (indicating possible mental disorder). As would be expected, women who showed mental disorders, scores greater than 63 per the SCL, had a lower score on the SF-36, meaning they had a decreased quality of life. This data suggests that psychiatric illness may go undetected in patients with PCOS. This data does not, however, suggest that the degree of emotional distress and reduced quality of life can be accurately predicted. ${ }^{32}$ On the other hand, this data shows that just because a woman has PCOS and anxiety/distress, it does not mean that she necessarily has a mental or psychiatric disorder. This further justifies the need for treatment of PCOS patients to be individualized.

Women with PCOS come from various backgrounds (culture, religion, socioeconomic status, and other comorbidities). Physicians and health care providers must ensure that they assess emotional distress on an individual basis. From here, providers can decide whether or not to further diagnosis or treatment by another health care specialist is required. Treating a PCOS patient should be multi-faceted and should involve a team of multiple medical experts. The extent to which physical symptoms have been correlated to psychological and emotional distress is limited and needs to be further explored. Although studies are scarce in this dimension, a majority of experts acknowledge that the psychosocial burden in women with PCOS cannot be ignored, and should be treated as a secondary symptom. ${ }^{17}$

\section{Hirsutism}

Hirsutism is often cited by patients as being one of the most disturbing aspects of PCOS, ${ }^{34}$ causing marked psychological stress. ${ }^{35}$ Hirsutism is the excessive hair growth in women following a male distribution pattern and can be evaluated through the Ferriman-Gallwey (FG) map scoring system.
The degree of hirsutism found in patients determined by the FG score is influenced not only by androgen levels but is also linked to insulin levels and insulin resistance. ${ }^{36,37}$ Therefore lifestyle changes may have a positive effect on the speed of hair growth, which can help improve QOL. Facial hair in women has been shown to cause distress. ${ }^{38}$ Not only does the distress stem from the hair growth itself, but from the considerable amount of time and energy to try to keep the condition hidden. ${ }^{35,39,40}$ Women with PCOS who experience hirsutism have often expressed that they feel "unfeminine," "freakish," "weird," and "different."41 This study interviewed 30 women with PCOS to find out that the three most troublesome problems for them were excess hair growth, irregular menses, and infertility. All three of these characteristics were areas women described as what they defined as being feminine and, as a result of PCOS, they felt robbed of their female identity. In this interview, patients were asked to describe their diagnosis with PCOS, symptoms they had and how they dealt with them, and also how they felt PCOS had influenced their lives. ${ }^{41}$

The feedback from these 30 women were eye opening to the difficulties that a women with hirsutism experiences. One interviewee commented, "A woman with a beard is, you know terrible. I think the hair is the worst thing because it's visible and it's so abnormal." Facial hair was a problem for $90 \%$ of patients who participated in the PCOSQ development group. ${ }^{16}$ From these numbers it is clear that this is an important issue for women with PCOS. Not only is excessive hair a concern, but the desire to get help has also been posed as a difficulty. Women expressed that they felt health care professionals did not take their symptoms seriously. ${ }^{41}$ Having a close and trusting relationship is crucial in the management of any syndrome and PCOS should not be any different. Health care providers need to make sure that their patients fully understand PCOS, and they are compassionate about the concerns patients may have.

Another study found that more severe hirsutism was associated with lower SF-36 scale scores for pain, general health perception, the physical sum scale, as well as decreased sexual self-worth and sexual satisfaction. ${ }^{8}$ Simultaneously, a study in Germany found that there could be no correlation between hirsutism and psychosocial distress or in areas of quality of life concerning emotional and social functioning. ${ }^{8}$ This study brings into question: Are there differences between hirsutism in different cultures? Limitations to this study also include the use of generic instruments. Researchers acknowledge that a disease specific instrument may be more sensitive to changes. ${ }^{8}$ Due to the limited amount of research 
in this area, health care professionals need to pay attention to the psychosocial dimension of PCOS on an individual basis, as it may not be possible to predict based on the presence or absence of symptoms such as hirsutism. ${ }^{8}$

\section{Metabolic syndrome and coronary artery disease}

Cardiovascular disease remains the leading cause of morbidity and mortality in women. ${ }^{42}$ Metabolic syndrome is a combination of cardiovascular disease risk factors associated with glucose intolerance, dyslipidemia, hypertension, and central obesity. ${ }^{11}$ Most associate PCOS with its ovulatory detriments, but research has shown growing concern in the area of metabolic syndrome and coronary artery disease in patients with PCOS. ${ }^{43,44}$ In fact, regardless of the diagnostic criteria used (NIH, Rotterdam or AES), fertile women with PCOS show an increase in prevalence of metabolic syndrome and hyperinsulinism compared with the healthy population; ${ }^{45}$ indicating the importance of metabolic parameters and insulin resistance in PCOS. As we know, PCOS can have debilitating aspects that affects a woman's day to day life. It is very important to treat the symptoms of PCOS such as hirsutism, infertility, and menstrual irregularity, but it is wise to think about the future of these patients and what other complications may stem from PCOS that affect the patient population. Complications such as $\mathrm{CAD}$, endometrial carcinoma, metabolic syndrome, sleep apnea, nonalcoholic steatohepatosis, depression, diabetes and breast cancer are some important things to keep in mind when facing a woman with PCOS.

It has been estimated that myocardial infarction is seven times more likely in patients with PCOS. ${ }^{46}$ It has also been observed that there is a $50 \%$ greater risk of myocardial infarction or fatal CHD among women with a history or irregular menstrual cycles at ages 20-35 years compared with a control group. ${ }^{47}$ PCOS can cause irregular menstrual cycles, so women with PCOS are in the population of women at higher risk for myocardial infarction. ${ }^{47}$ Additionally, cardiac catheterization studies have shown more extensive coronary artery disease in patients with PCOS compared to that of healthy control groups. ${ }^{48}$ These findings are alarming, and justify the need for further investigation into the several dimensions of PCOS. Not only have several coronary risk factors been well documented in women with PCOS, but elevated rates of obesity, ${ }^{46,49,50}$ central obesity, ${ }^{49}$ glucose intolerance ${ }^{11,51}$ and increased blood pressure ${ }^{49}$ have been found.

A 2006 study found that of 78 adolescents with PCOS had a substantially higher risk of developing metabolic syndrome and hypertension compared with the general female adolescent population; this information was obtained from the third National Health and Nutrition Examination Survey (NHANES III) using the Cook criteria (37\% vs 5\%) and the de Ferranti criteria (47\% vs 13\%). ${ }^{52,53}$ This study also had other interesting findings; none of the girls of normal body mass index had metabolic syndrome, whereas $11 \%$ of overweight and $63 \%$ of obese girls with PCOS had metabolic syndrome compared with 0 and $32 \%$ of the NHANES III population. ${ }^{11}$ In total, of the 78 adolescent girls with PCOS, $63 \%$ of them had the complete data for diagnosis of metabolic syndrome.

In general, studies support the trend that PCOS women are at higher risk for metabolic syndrome and have an increased risk for cardiovascular diseases even with small sample sizes. It should be taken into account that different studies use different criteria, often not making the populations between studies homogenous. ${ }^{42}$ Additionally, many studies take place in different areas of the world making it hard to generalize information to fit all women with PCOS. For example, it was found that the prevalence of metabolic syndrome in PCOS patients appears to be much lower in Italy than in the United States. ${ }^{54}$ Race is also an important factor when performing these studies, and not all studies have included this information. A 2006 study showed that among women with PCOS, whites, blacks, and Hispanics were more likely to be obese; Asians and Hispanics were more likely to have diabetes; and blacks were more likely to have hypertension, while Hispanics were less likely to have hypertension. ${ }^{55}$ It is not accurate to say that current literature in regards to metabolic syndrome in PCOS has not provided useful information, but continued studies are needed. Studies such as one with large sample size comparing women not just to control groups but also to women of different cultures, is needed to help make suggestions to the public on this disorder.

Research has shown that keeping PCOS under control and preventing components of metabolic syndrome will work if the physician and patient work together. Physicians must provide early intervention and long-term monitoring for women with PCOS and need to continue this lifelong monitoring; these patients are at increased risk for complications as they age, as body mass index increases, and in the presence of diabetes. ${ }^{56,57}$ Early treatment is the key with these patients, no matter their age at diagnosis. Even young, obese adolescents with PCOS have been found to have a high prevalence of early endocrine, metabolic, and cardiovascular characteristics. ${ }^{2}$ Many early symptoms of coronary artery diseases are silent, making it important to 
keep up with routine lab tests and monitoring. Patients must also take responsibility in their health care and make lifestyle modifications such as diet, exercise, and smoking cessation to help prevent complications of metabolic syndrome. ${ }^{57}$

Obesity and diabetes have increased dramatically worldwide during the last decade. ${ }^{56}$ Losing weight, if overweight, is a critical part of a woman's health, especially those with PCOS. ${ }^{58}$ Not only does obesity act as a risk factor for other health problems, it has been shown that obesity and weight gain are likely to lead to loss of self-esteem and poor body image, resulting in a decreased quality of life and psychological morbidity. ${ }^{19}$ If a woman is overweight, oral glucose tests should be performed by the patient's physician. It has been found that greater than $25 \%$ of obese women with PCOS will develop impaired glucose or type 2 diabetes ${ }^{57}$ For physicians, it is important to stress to patients with PCOS that the best therapy for them is weight loss; hyperinsulinemia and hyperandrogenism can be reduced with weight loss. ${ }^{57}$ It has been well documented that insulin resistance is responsible for the high androgen concentration in women with PCOS. ${ }^{59}$ Not only could losing weight help women prevent metabolic complications, but it can aid in decreasing their symptoms of hyperandrogenism, such as excessive hair growth and acne, and possibly increase their quality of life. It is also important to remember that in PCOS patients' lipid metabolism is often impaired. In patients who are obese, it has been shown that there is a specific reduction in high-density lipoprotein lipid formation, therefore a reduced capacity for cholesterol elimination. ${ }^{58}$ One study has shown that there are some metabolic features of PCOS that are present in daughters of PCOS women before the onset of hyperandrogenism, so not only is it important to care for their own bodies, but also for the future of their daughters. ${ }^{60,61}$

\section{Conclusion}

PCOS is not just an endocrine disorder, but a combination of metabolic and psychosocial detriments. Not addressing all the aspects of PCOS, such as depression, may delay the treatment of the "primary" issues such as fertility and hyperandrogenism. While further research needs to be conducted with larger sample sizes, current research does suggest a decreased quality of life in this population. It is evident that a universally accepted diagnostic criterion needs to be implemented by researchers and health care professionals. The longer proper treatment is delayed, chances are increased of the patient developing other health issues such as metabolic syndrome and cardiovascular disease. Treating the patient holistically seems like a very simple answer to a large problem; this will only happen if health care professionals are committed to increasing patient education, and helping patients find the proper treatment for all aspects of this disease.

\section{Disclosure}

The authors report no conflicts of interest in this work.

\section{References}

1. Krysiak R, Okopien B, Gdula-Dymek A, Herman ZS. Update on the management of polycystic ovary syndrome. Pharmacol Rep. 2006;58:614-625.

2. Shroff R, Kerchner A, Maifeld M, Van Beek EJ, Jagasia D, Dokras A. Young obese women with polycystic ovary syndrome have evidence of early coronary atherosclerosis. J Clin Endocrinol Metab. 2007; 92:4609-4614.

3. Jones GL, Benes K, Clark TL, et al. The Polycystic Ovary Syndrome Health-Related Quality of Life Questionnaire (PCOSQ): a validation. Hum Reprod. 2004;19:371-377.

4. Torpy JM, Lynm C, Glass RM. JAMA patient page. Polycystic ovary syndrome. JAMA. 2007;297:554.

5. Broekmans FJ, Knauff EA, Valkenburg O, Laven JS, Eijkemans MJ, Fauser BC. PCOS according to the Rotterdam consensus criteria: Change in prevalence among WHO-II anovulation and association with metabolic factors. BJOG. 2006;113:1210-1217.

6. Stankiewicz M, Norman R. Diagnosis and management of polycystic ovary syndrome: a practical guide. Drugs. 2006;66:903-912.

7. Azziz R, Carmina E, Dewailly D, et al. Criteria for defining polycystic ovary syndrome as a predominantly hyperandrogenic syndrome: an androgen excess society guideline. J Clin Endocrinol Metab. 2006;91:4237-4245.

8. Hahn S, Janssen OE, Tan S, et al. Clinical and psychological correlates of quality-of-life in polycystic ovary syndrome. Eur $J$ Endocrinol. 2005; $153: 853-860$.

9. Talbott EO, Guzick DS, Sutton-Tyrrell K, et al. Evidence for association between polycystic ovary syndrome and premature carotid atherosclerosis in middle-aged women. Arterioscler Thromb Vasc Biol. 2000;20:2414-2421.

10. Vural B, Caliskan E, Turkoz E, Kilic T, Demirci A. Evaluation of metabolic syndrome frequency and premature carotid atherosclerosis in young women with polycystic ovary syndrome. Hum Reprod. 2005;20:2409-2413.

11. Coviello AD, Legro RS, Dunaif A. Adolescent girls with polycystic ovary syndrome have an increased risk of the metabolic syndrome associated with increasing androgen levels independent of obesity and insulin resistance. J Clin Endocrinol Metab. 2006;91:492-497.

12. McCook JG, Reame NE, Thatcher SS. Health-related quality of life issues in women with polycystic ovary syndrome. J Obstet Gynecol Neonatal Nurs. 2005;34:12-20.

13. Elsenbruch S, Hahn S, Kowalsky D, et al. Quality of life, psychosocial well-being, and sexual satisfaction in women with polycystic ovary syndrome. J Clin Endocrinol Metab. 2003;88:5801-5807.

14. Coffey S, Bano G, Mason HD. Health-related quality of life in women with polycystic ovary syndrome: a comparison with the general population using the Polycystic Ovary Syndrome Questionnaire (PCOSQ) and the Short Form-36 (SF-36). Gynecol Endocrinol. 2006;22:80-86.

15. Streiner DL, Norman G. Health Measurement Scales: A practical guide to their development and use. 2nd ed. Oxford, UK: Oxford University Press; 2000.

16. Cronin L, Guyatt G, Griffith L, et al. Development of a health-related quality-of-life questionnaire (PCOSQ) for women with polycystic ovary syndrome (PCOS). J Clin Endocrinol Metab. 1998;83:1976-1987.

17. Eggers $\mathrm{S}$, Kirchengast $\mathrm{S}$. The polycystic ovary syndrome - a medical condition but also an important psychosocial problem. Coll Antropol. 2001;25:673-685. 
18. Schmid J, Kirchengast S, Vytiska-Binstorfer E, Huber J. Infertility caused by PCOS - health-related quality of life among Austrian and Moslem immigrant women in Austria. Hum Reprod. 2004;19:2251-2257.

19. Ching HL, Burke V, Stuckey BG. Quality of life and psychological morbidity in women with polycystic ovary syndrome: body mass index, age and the provision of patient information are significant modifiers. Clin Endocrinol (Oxf). 2007;66:373-379.

20. Homburg R. What is polycystic ovarian syndrome? A proposal for a consensus on the definition and diagnosis of polycystic ovarian syndrome. Hum Reprod. 2002;17:2495-2499.

21. Goldzieher JW, Axelrod LR. Clinical and biochemical features of polycystic ovarian disease. Fertil Steril. 1963;14:631-653.

22. Paulson JD, Haarmann BS, Salerno RL, Asmar P. An investigation of the relationship between emotional maladjustment and infertility. Fertil Steril. 1988;49:258-262.

23. Downey J, Yingling S, McKinney M, Husami N, Jewelewicz R, Maidman J. Mood disorders, psychiatric symptoms and distress in women presenting for infertility evaluation. Fertil Steril. 1989;52:425-432.

24. Azziz R, Marin C, Hoq L, Badamgarav E, Song P. Health carerelated economic burden of the polycystic ovary syndrome during the reproductive life span. J Clin Endocrinol Metab. 2005;90:4650-4658.

25. Monga M, Alexandrescu B, Katz SE, Stein M, Ganiats T. Impact of infertility on quality of life, marital adjustment, and sexual function. Urology. 2004;63:126-130.

26. Boivin J, Takefman JE, Tulandi T, Brender W. Reactions to infertility based on extent of treatment failure. Fertil Steril. 1995;63:801-807.

27. Guerra D, Llobera A, Veiga A, Barri PN. Psychiatric morbidity in couples attending a fertility service. Hum Reprod. 1998;13:1733-1736.

28. Oddens BJ, den Tonkelaar I, Nieuwenhuyse H. Psychosocial experiences in women facing fertility problems - a comparative survey. Hum Reprod. 1999;14:255-261.

29. Fekkes M, Buitendijk SE, Verrips GH, et al. Health-related quality of life in relation to gender and age in couples planning IVF treatment Hum Reprod. 2003;18:1536-1543.

30. Esler DM, Travers CA, Guttikonda K, Dixon A, Lewis PR. The psychosocial experience of women with PCOS - a case control study. Aust Fam Physician. 2007;36:965-967.

31. Weiner CL, Primeau M, Ehrmann DA. Androgens and mood dysfunction in women: comparison of women with polycystic ovarian syndrome to healthy controls. Psychosom Med. 2004;66:356-362.

32. Elsenbruch S, Benson S, Hahn S, et al. Determinants of emotional distress in women with polycystic ovary syndrome. Hum Reprod. 2006;21:1092-1099.

33. Derogatis LR. SCL-90-R: Administration, Scoring and Procedures Manual II. Baltimore, MD: Clinical Psychometric Research; 1983.

34. Coffey S, Mason H. The effect of polycystic ovary syndrome on healthrelated quality of life. Gynecol Endocrinol. 2003;17:379-386.

35. Sonino N, Fava GA, Mani E, Belluardo P, Boscaro M. Quality of life of hirsute women. Postgrad Med J. 1993;69:186-189.

36. Amato MC, Galluzzo A, Merlino S, et al. Lower insulin sensitivity differentiates hirsute from non-hirsute Sicillian women with polycystic ovary syndrome. Eur J Endocrinol. 2006;155:859-865.

37. Landay M, Huang A, Azziz R. Degree of hyperinsulinemia, independent of androgen levels, is an important determinant of the severity of hirsutism in PCOS. Fertil Steril. 2008;Aug 21 [Epub ahead of print].

38. Rabinowitz S, Cohen R, Le Roith D. Anxiety and hirsutism. Psychol Rep. 1983;53(3 Pt 1):827-830.

39. Conn JJ, Jacobs HS. Managing hirsutism in gynaecological practice. Br J Obstet Gynaecol. 1998;105:687-696.

40. Lanigan S, Kwan C, Dykes P, Gonzales M. Quality of life studies in hirsute women receiving ruby laser treatment. $\mathrm{Br} J$ Dermatol. 2000;143(Suppl 57):50.

41. Kitzinger C, Willmott J. 'The thief of womanhood': women's experience of polycystic ovarian syndrome. Soc Sci Med. 2002;54:349-361.
42. Legro RS. Polycystic ovary syndrome and cardiovascular disease: a premature association? Endocr Rev. 2003;24:302-312.

43. Apridonidze T, Essah PA, Iuorno MJ, Nestler JE. Prevalence and characteristics of the metabolic syndrome in women with polycystic ovary syndrome. J Clin Endocrinol Metab. 2005;90:1929-1935.

44. Ehrmann DA, Liljenquist DR, Kasza K, Azziz R, Legro RS, Ghazzi MN. Prevalence and predictors of the metabolic syndrome in women with polycystic ovary syndrome. J Clin Endocrinol Metab. 2006;91:48-53.

45. Amato MC, Galluzzo A, Finocchiaro S, Criscimanna A, Giordano C. The evaluation of metabolic parameters and insulin sensitivity for a more robust diagnosis of the polycystic ovary syndrome. Clin Endocrinol (Oxf). 2008;69(1):52-60.

46. Dahlgren E, Janson PO, Johansson S, Lapidus L, Oden A. Polycystic ovary syndrome and risk for myocardial infarction. Evaluated from a risk factor model based on a prospective population study of women. Acta Obstet Gynecol Scand. 1992;71:599-604.

47. Solomon CG, Hu FB, Dunaif A, et al. Menstrual cycle irregularity and risk for future cardiovascular disease. J Clin Endocrinol Metab. 2002;87:2013-2017.

48. Birdsall MA, Farquhar CM, White HD. Association between polycystic ovaries and extent of coronary artery disease in women having cardiac catheterisation. Ann Intern Med. 1997;126:32-35.

49. Talbott E, Guzick D, Clerici A, et al. Coronary heart disease risk factors in women with polycystic ovary syndrome. Arterioscler Thromb Vasc Biol. 1995;15:821-826.

50. Wild S, Pierpoint T, McKeigue P, Jacobs H. Cardiovascular disease in women with polycystic ovary syndrome at long-term follow-up: a retrospective cohort study. Clin Endocrinol (Oxf). 2000;52:595-600.

51. Montague CT, O'Rahilly S. The perils of portliness: causes and consequences of visceral adiposity. Diabetes. 2000;49:883-888.

52. Cook S, Weitzman M, Auinger P, Nguyen M, Dietz WH. Prevalence of a metabolic syndrome phenotype in adolescents:findings from the thrid National Health and Nutrition Examination Survey, 1988-1994. Arch Pediatr Adolesc Med. 2003;157:821-827.

53. de Ferranti SD, Gauvreau K, Ludwig DS, Neufeld EJ, Newburger JW, Rifai N. Prevalence of the metabolic syndrome in American adolescents: findings from the Third National Health and Nutrition Examination Survey. Circulation. 2004;110:2494-2497.

54. Carmina E, Napoli N, Longo RA, Rini GB, Lobo RA. Metabolic syndrome in polycystic ovary syndrome (PCOS): lower prevalence in southern Italy than in the USA and the influence of criteria for the diagnosis of PCOS. Eur J Endocrinol. 2006;154:141-145.

55. Lo JC, Feigenbaum SL, Yang J, Pressman AR, Selby JV, Go AS Epidemiology and adverse cardiovascular risk profile of diagnosed polycystic ovary syndrome. J Clin Endocrinol Metab. 2006;91: 1357-1363.

56. Lauenborg J, Mathiesen E, Hansen T, et al. The prevalence of the metabolic syndrome in a Danish population of women with previous gestational diabetes mellitus is three-fold higher than in the general population. J Clin Endocrinol Metab. 2005;90:4004-4010.

57. Vignesh JP, Mohan V. Polycystic ovary syndrome: a component of metabolic syndrome? J Postgrad Med. 2007;53:128-134.

58. Rajkhowa M, Neary RH, Kumpatla P, et al. Altered composition of high density lipoproteins in women with the polycystic ovary syndrome. J Clin Endocrinol Metab. 1997;82:3389-3394.

59. Kazerooni T, Dehghan-Kooshkghazi M. Effects of metformin therapy on hyperandrogenism in women with polycystic ovarian syndrome. Gynecol Endocrinol. 2003;17:51-56.

60. Leibel NI, Baumann EE, Kocherginsky M, Rosenfield RL. Relationship of adolescent polycystic ovary syndrome to parental metabolic syndrome. J Clin Endocrinol Metab. 2006;91:1275-1283.

61. Sir-Petermann T, Maliqueo M, Codner E, et al. Early metabolic derangements in daughters of women with polycystic ovary syndrome. J Clin Endocrinol Metab. 2007;92:4637-4642. 
